\title{
Comparative effectiveness of exercise and drug interventions on mortality outcomes: metaepidemiological study
}

\author{
(c) $\frac{(1)(8)}{\text { gy }}$ OPEN ACCESS
}

\author{
Huseyin Naci researcher ${ }^{1}$ fellow ${ }^{2}$, John P A loannidis director ${ }^{3}$
}

'LSE Health, London School of Economics and Political Science, London, UK; ${ }^{2}$ Drug Policy Research Group, Department of Population Medicine, Harvard Medical School and Harvard Pilgrim Health Care Institute, Boston, MA, USA; ${ }^{3}$ Stanford Prevention Research Center, Stanford University School of Medicine, Stanford, CA, USA

\begin{abstract}
Objective To determine the comparative effectiveness of exercise versus drug interventions on mortality outcomes.

Design Metaepidemiological study.

Eligibility criteria Meta-analyses of randomised controlled trials with mortality outcomes comparing the effectiveness of exercise and drug interventions with each other or with control (placebo or usual care).
\end{abstract}

Data sources Medline and Cochrane Database of Systematic Reviews, May 2013

Main outcome measure Mortality.

Data synthesis We combined study level death outcomes from exercise and drug trials using random effects network meta-analysis.

Results We included 16 (four exercise and 12 drug) meta-analyses. Incorporating an additional three recent exercise trials, our review collectively included 305 randomised controlled trials with 339274 participants. Across all four conditions with evidence on the effectiveness of exercise on mortality outcomes (secondary prevention of coronary heart disease, rehabilitation of stroke, treatment of heart failure, prevention of diabetes), 14716 participants were randomised to physical activity interventions in 57 trials. No statistically detectable differences were evident between exercise and drug interventions in the secondary prevention of coronary heart disease and prediabetes. Physical activity interventions were more effective than drug treatment among patients with stroke (odds ratios, exercise $v$ anticoagulants $0.09,95 \%$ credible intervals 0.01 to 0.70 and exercise $v$ antiplatelets $0.10,0.01$ to 0.62 ). Diuretics were more effective than exercise in heart failure (exercise $v$ diuretics $4.11,1.17$ to 24.76 ). Inconsistency between direct and indirect comparisons was not significant.

Conclusions Although limited in quantity, existing randomised trial evidence on exercise interventions suggests that exercise and many drug interventions are often potentially similar in terms of their mortality benefits in the secondary prevention of coronary heart disease, rehabilitation after stroke, treatment of heart failure, and prevention of diabetes.

\section{Introduction}

Physical activity has well documented health benefits. ${ }^{1}$ Population level cohort studies have shown that people who exercise enjoy a higher quality of life and improved health status compared with those with sedentary behaviours, with subsequent reductions in their risk of adverse outcomes such as admissions to hospital. Randomised controlled trials have shown similarly favourable findings in arthritis, ${ }^{2}$ cancer, ${ }^{34}$ diabetes, ${ }^{5}$ heart disease, ${ }^{6}$ and respiratory illnesses, ${ }^{7}$ among other chronic conditions. ${ }^{8} 9$ Large scale observational studies have also established a clear association between exercise and all cause mortality. ${ }^{10-12}$ Given the overwhelming evidence in support of the health benefits of exercise, ${ }^{13}$ the Global Burden of Disease study has recently ranked physical inactivity as the fifth leading cause of disease burden in western Europe, and as one of the top modifiable risk factors along with smoking. ${ }^{14}$

Despite recent calls to encourage physical activity as a strategy to ward off the emerging burden of chronic conditions, including heart disease and diabetes, ${ }^{15}$ population level physical activity measures are discouraging. In the United Kingdom, only 14\% of adults exercise regularly, with roughly one third of adults in England meeting recommended levels of physical activity. ${ }^{16}$ In contrast, utilisation rates of prescription drugs continue to rise sharply, increasing to an average of 17.7 prescriptions for every person in England in 2010, compared with 11.2 in $2000 .^{17}$

Abundant evidence from randomised controlled trials shows the mortality benefits of certain drugs such as simvastatin in the secondary prevention of cardiovascular disease, ${ }^{18}$ which is 
the most widely prescribed drug in the United Kingdom. Research on the mortality benefits of exercise, however, remains primarily observational with a limited number of randomised trials in select treatment areas. ${ }^{19}$ More importantly, evidence on how physical activity interventions fare compared with drug interventions in reducing the risk of all cause mortality is lacking.

We performed a comprehensive review of published meta-analyses on topics with randomised trial evidence on both exercise and drug interventions. For each condition, we combined the data from multiple pairwise meta-analyses in network meta-analyses to assess the geometry of the existing evidence and to determine the comparative effectiveness of drug and exercise interventions in reducing the risk of mortality.

\section{Methods}

\section{Identification of relevant evidence}

We used three steps to identify the relevant body of evidence. Firstly, we searched Medline for meta-analyses of randomised controlled trials evaluating the effectiveness of exercise based interventions on mortality outcomes. Our search strategy included terms for exercise interventions (exercise OR physical activity), mortality outcomes (mortality OR death OR survival), and meta-analysis of randomised controlled trials (randomized OR meta-analysis) in the title, abstract, or keywords (latest search 11 December 2012).

Secondly, for all conditions with evidence on the effectiveness of exercise interventions on mortality outcomes, we identified meta-analyses that evaluated the impact of specific drug interventions on mortality outcomes. We identified the list of relevant drug interventions using clinical practice guidelines, developed by respective European and US clinical specialty organisations (for example, American Heart Association, European Society of Cardiology). In cases where clinical practice guidelines did not give clear guidance around indicated drug treatments, we consulted the US National Library of Medicine's Medline Plus website (www.nlm.nih.gov/ medlineplus/). For each drug of interest we searched the Cochrane Database of Systematic Reviews to identify the most up to date meta-analysis. In cases where Cochrane reviews did not exist, we searched Medline using terms for drug names, mortality outcomes (mortality OR death OR survival), and meta-analysis of randomised controlled trials (randomized OR meta-analysis).

Finally, we developed a separate search strategy in Medline to identify randomised head to head comparisons of exercise versus drug interventions (and their meta-analyses) for all conditions with evidence on the effectiveness of exercise interventions on mortality outcomes. The objective of this search was to capture additional recent trials of exercise versus control intervention that were not included in the meta-analyses evaluating the effectiveness of exercise based interventions on mortality outcomes. This search included terms for exercise interventions (exercise OR physical activity), mortality outcomes (mortality OR death OR survival), and Cochrane Collaboration's sensitivity and precision maximising terms for randomised controlled trials (latest search 27 May 2013). ${ }^{20}$

In all three steps we selected the most recent review if more than one eligible meta-analysis was published for a given condition or disease and for each particular intervention. We considered studies embedded within meta-analyses (or single studies identified in the third step) to be eligible for inclusion if they were randomised controlled trials comparing the effectiveness of exercise and drug interventions with another or with control (placebo or usual care) on mortality outcomes.

\section{Data extraction}

From each eligible trial we extracted the publication year, condition, number of participants, and number of deaths in each trial arm. In cases where mortality outcomes were reported at multiple time points, we selected the longest follow-up duration. One investigator $(\mathrm{HN})$ extracted data and another (Anthony J Damico) checked for accuracy.

\section{Evaluation of the geometry of evidence}

We developed network diagrams to visualise the geometry of the available evidence ${ }^{21} 22$; that is, the frequency of comparisons between exercise and drug interventions to show the distribution of trial participants across different treatment modalities (and control). In each network diagram, the size of the nodes was proportional to the number of participants receiving a given intervention (or control), and the thickness of the lines connecting the nodes was proportional to the number of randomised participants in the trials between interventions. We developed two sets of network diagrams. In the first we compared exercise to all identified classes of drugs. In the second we pooled all drug interventions into one group to show the relative size of the patient population included in the trials of exercise versus drug interventions.

\section{Statistical analysis}

We first qualitatively summarised included trials, describing the types of direct and indirect comparisons. For each direct comparison between two treatments we conducted pairwise meta-analyses using the DerSimonian-Laird (random effects) method, ${ }^{23}$ and statisticaly inspected heterogeneity using the $\mathrm{I}^{2}$ measure. We used rough $\mathrm{I}^{2}$ thresholds of $25 \%, 50 \%$, and $75 \%$ to define low $(<25 \%)$, moderate $(25-49 \%)$, high $(50-75 \%)$, and very high $(>75 \%)$ heterogeneity. To determine the comparative effectiveness of exercise and drug interventions, we then conducted network meta-analysis, which is a generalisation of indirect comparisons across pairwise meta-analyses. ${ }^{24}$ This type of analysis allows for comparing interventions in the absence of head to head trials comparing all the interventions of interest. ${ }^{25}$ In cases where both direct (from trials that include a specific pairwise comparison) and indirect (from a network of trials that do not include that comparison) sources of evidence exist, network meta-analysis is capable of simultaneously combining both types of evidence. This analysis preserves the within trial randomised treatment comparison of each trial while combining all available comparisons between treatments.

We combined study level relative treatment effects using bayesian Markov chain Monte Carlo methods in WinBUGS version 1.4.3. We used the model developed by Dias and colleagues for the UK National Institute for Health and Care Excellence Decision Support Unit. ${ }^{26}{ }^{27}$ Our models adopted random effects, taking into account potential heterogeneity by assuming that each treatment was drawn from the same distribution, the mean and variance of which were estimated from the data. ${ }^{28} \mathrm{We}$ also performed fixed effect analyses under the assumption that no between study heterogeneity existed. ${ }^{29}$ Our model selection was based on two criteria. Firstly, we evaluated the deviance information criterion between the two sets of analyses, which favoured random effects models. Secondly, we qualitatively judged the fixed effect model assumption to be too strong given the potential differences in patient populations across drug and exercise trials. ${ }^{25}$ 
We statistically evaluated the consistency of direct and indirect evidence in network meta-analyses using the Bucher method. ${ }^{30}$ This method, commonly used to investigate potential inconsistency in evidence networks, ${ }^{31} 32$ was based on conducting random effects pairwise meta-analyses on all comparisons and calculating the difference in log odds ratios between direct and indirect estimates for each closed loop of the network (that is, in all instances where both direct and indirect comparisons could be generated for each contrast in the network).

We reported our findings in terms of odds ratios. The difference between treatments was assessed on the basis of $95 \%$ credible intervals. Such intervals can be interpreted as indicating a $95 \%$ probability that the true odds ratio falls within the reported range. If a $95 \%$ credible interval does not include the null value 1.00 , this can be interpreted as indicating $<5 \%$ probability that there is no difference between the two comparators. Given the bayesian nature of the statistical analyses, we neither estimated nor reported $\mathrm{P}$ values for the network meta-analyses.

Primary analyses were at the intervention level, comparing exercise with all relevant drug interventions for a given condition. In a separate analysis we also pooled the drugs and estimated the comparative effectiveness of exercise versus all drug interventions. The analyses were based on the total number of participants per trial arm, as reported in the identified meta-analyses.

\section{Results \\ Eligible meta-analyses}

Our electronic search of exercise meta-analyses retrieved 225 titles and abstracts (fig $1 \Downarrow$ ). After exclusions, we included four meta-analyses of exercise interventions reporting mortality outcomes (see supplementary table). One meta-analysis evaluated the effectiveness of exercise interventions for the secondary prevention of coronary heart disease, ${ }^{33}$ one for stroke, ${ }^{34}$ one for heart failure, ${ }^{35}$ and one for prediabetes. ${ }^{36}$

In addition we separately identified 12 meta-analyses of drug interventions (out of 534 titles and abstracts in Medline), which were considered to be relevant drug options for each of the four conditions that had evidence on exercise interventions: statins, ${ }^{18}$ $\beta$ blockers, ${ }^{37}$ angiotensin converting enzyme inhibitors, ${ }^{38}$ and antiplatelets for the secondary prevention of coronary heart disease $^{39}$; anticoagulants ${ }^{40}$ and antiplatelets ${ }^{41}$ for stroke ${ }^{42}$; angiotensin converting enzyme inhibitors, ${ }^{43}$ diuretics, ${ }^{44} \beta$ blockers, ${ }^{45}$ and angiotensin receptor blockers for heart failure ${ }^{46}$; $\alpha$ glucosidase inhibitors, thiazolidinediones, biguanides, angiotensin converting enzyme inhibitors, and glinides for prediabetes. ${ }^{36}$ Publication dates for meta-analyses of drug interventions ranged from 1999 to 2013 (see supplementary table).

Most of the meta-analyses of drug interventions were placebo controlled. However, one meta-analysis included direct head to head trials comparing anticoagulants with antiplatelets for the treatment of stroke, ${ }^{47}$ and one meta-analysis included a direct comparison between angiotensin receptor blockers and angiotensin converting enzyme inhibitors for the treatment of heart failure. ${ }^{46}$ Similarly, the meta-analysis on prediabetes included not only the placebo controlled trials of drug and exercise interventions but also their direct head to head comparisons. ${ }^{36}$

In total, our original search identified 16 (four exercise and 12 drug) meta-analyses, including 54 exercise trials and 248 drug trials. Our separate electronic search in Medline, which yielded 2964 abstracts and titles, did not identify any additional direct head to head trials of exercise and drug interventions evaluating mortality outcomes for coronary heart disease, stroke, heart failure, and prediabetes. This search captured 31 of the 54 exercise trials that were already included in the four exercise meta-analyses. It also captured another three recent trials of exercise versus control intervention published between 2008 and 2012 (total sample size 2041) that had not been included in the four meta-analyses. Including these three exercise trials in the analysis, our meta-epidemiological review collectively included 305 randomised controlled trials with 339274 participants. Of those, 57 trials concerned exercise interventions, including 14716 participants.

\section{Characteristics of exercise interventions}

The characteristics of the exercise interventions varied across treatment areas. Differences included the mode of physical activity and its frequency, intensity, and duration. Exercise based cardiac rehabilitation was typically a component of comprehensive cardiac care of patients with coronary heart disease. ${ }^{33}$ Rehabilitation in this population included inpatient, outpatient, community based, or home based exercise interventions. Patients with stroke received a mix of cardiorespiratory and muscle strengthening exercises. ${ }^{34}$ Similarly, exercise interventions targeting patients with chronic heart failure included aerobic and resistance training. ${ }^{35}$ Physical activity was often a component of multifactorial lifestyle modification interventions to prevent diabetes among people with impaired glucose tolerance and impaired fasting glucose-that is, prediabetes. ${ }^{36}$

\section{Comparability of trial and patient population characteristics}

Conceptual heterogeneity between drug and exercise trials was minimal among patients with coronary heart disease (about $60-100 \%$ of patients had myocardial infarctions), heart failure (patients were predominantly New York Heart Association class II-IV), and prediabetes (patients had impaired glucose tolerance and impaired fasting glucose with a balanced profile of obesity and cardiovascular disease risk factors across exercise and drug trials). However, the baseline disease severity of patient populations was considerably different in stroke trials. While survivors participating in drug trials were within two weeks of onset of stroke, patients included in exercise trials were predominantly ambulatory and could be up to 150 days after stroke.

In all four conditions, trial characteristics varied for eligibility criteria, follow-up duration, and blinding of participants, investigators, and assessors (see supplementary table). In general, variability within pairwise meta-analyses was greater than between exercise and drug interventions. Statistical variability in effect sizes was low in the majority of pairwise meta-analyses, with moderate between study heterogeneity present in the trials of angiotensin converting enzyme inhibitors in the secondary prevention of coronary heart disease $\left(\mathrm{I}^{2}=40 \%\right)$, trials of $\beta$ blockers in heart failure $\left(\mathrm{I}^{2}=34 \%\right)$, and trials comparing angiotensin receptor blockers with angiotensin converting enzyme inhibitors in heart failure $\left(I^{2}=39 \%\right)$.

\section{Geometry of available evidence}

In all four conditions, relatively few participants were randomised to exercise interventions compared with the available evidence on drugs. Thirty four trials concerned exercise interventions in the secondary prevention of coronary heart disease ( $n=10984$ participants) compared with 43 trials of statins 
$(n=55151), 80$ of $\beta$ blockers $(n=53456), 15$ of angiotensin converting enzyme inhibitors ( $\mathrm{n}=15$ 104), and 27 of antiplatelets $(n=38076)$. No direct head to head trials compared any of the drug or exercise interventions in the identified meta-analyses, resulting in a star shaped evidence network for the secondary prevention of coronary heart disease (fig $2 \Downarrow$ ).

Three trials concerned exercise interventions among patients with stroke $(n=227)$ compared with 10 trials of anticoagulants $(n=22786), 14$ of antiplatelets $(n=43041)$, and three directly comparing anticoagulants with antiplatelets $(\mathrm{n}=11567$, fig 2$)$.

In the case of heart failure, 18 trials concerned exercise interventions $(\mathrm{n}=3669)$ compared with five trials of angiotensin converting enzyme inhibitors $(n=12763)$, three of diuretics $(n=202), 21$ of $\beta$ blockers $(n=23122), 11$ of angiotensin receptor blockers $(n=11795)$, and eight directly comparing angiotensin receptor blockers with angiotensin converting enzyme inhibitors (fig 2).

Two trials were of exercise interventions among participants with prediabetes ( $\mathrm{n}=1081$ participants). In comparison, one trial was of $\alpha$ glucosidase inhibitors $(n=1368)$, one of thiazolidinediones $(\mathrm{n}=407)$, two of angiotensin converting enzyme inhibitors $(n=14575)$, and one of glinides $(n=9306)$. One trial directly compared exercise with $\alpha$ glucosidase inhibitors ( $\mathrm{n}=1778)$, and two multiarm trials including 3615 participants compared exercise, biguanides, and control (fig 2).

When the drug trials were pooled, 5685 participants with coronary heart disease were randomised to exercise compared with 85421 randomised to control and 81655 to drug interventions (fig $3 \Downarrow$ ). In a similar fashion, considerably fewer participants were randomised to physical activity interventions in stroke trials $(\mathrm{n}=117)$, heart failure trials $(\mathrm{n}=1830)$, and prediabetes trials $(n=2491)$. Respective numbers of participants randomised to drug interventions were 44731,30 024, and 14 867.

\section{Comparative effectiveness of exercise and drugs}

In coronary heart disease the odds of mortality was reduced with use of statins (odds ratio $0.82,95 \%$ credible interval 0.75 to 0.90$), \beta$ blockers $(0.85,0.78$ to 0.92$)$, angiotensin converting enzyme inhibitors $(0.83,0.72$ to 0.96$)$, and antiplatelets $(0.83$, 0.74 to 0.93 ) compared with control, whereas exercise interventions had a similar point estimate but wider credible intervals that extended on both sides of 1.00 (odds ratio 0.89 , $95 \%$ credible interval 0.76 to 1.04 , fig $4 \Downarrow$ ). When compared head to head in network meta-analyses, all interventions were not different beyond chance: there were no statistically detectable differences among any of the exercise and drug interventions in terms of their effects on mortality outcomes (tables $1 \Downarrow$ ).

Unlike any of the drug interventions, exercise was significantly more effective than control in reducing the odds of mortality $(0.09,0.01$ to 0.72$)$ among patients with stroke (fig 4). When compared head to head in network meta-analyses, exercise interventions were more effective than anticoagulants $(0.09$, 0.01 to 0.70$)$ and antiplatelets $(0.10,0.01$ to 0.62$)$, albeit with considerable uncertainty owing to the small number of events in exercise trials (table $2 \Downarrow$ ). Anticoagulants were also marginally worse than antiplatelets $(1.11,1.00$ to 1.21$)$.

In heart failure, fewer deaths occurred with diuretics $(0.19,0.03$ to 0.66$)$ and $\beta$ blockers $(0.71,0.61$ to 0.80$)$ compared with control (fig 3$)$. Diuretics were more effective than exercise $(0.24$, 0.04 to 0.85$)$, angiotensin converting enzyme inhibitors ( 0.21 , $0.03,0.76), \beta$ blockers $(0.27,0.04$ to 0.93$)$, and angiotensin receptor blockers $(0.21,0.03$ to 0.73 , table $3 \Downarrow)$. Angiotensin receptor blockers were associated with more deaths compared with $\beta$ blockers (1.30, 1.02 to 1.61 ).

Neither exercise nor drug interventions were clearly effective in reducing the odds of mortality in prediabetes (fig 4). There were also no major detectable differences between any comparator interventions (table $4 \Downarrow$ ).

In secondary analyses comparing exercise with the drug interventions pooled together, there was no definitive differences between drug and exercise interventions in coronary heart disease, heart failure, and prediabetes, but effect sizes had modestly substantial uncertainty for heart failure and prediabetes (table $5 \Downarrow$ ). Although exercise interventions were more effective than drugs in reducing the odds of mortality among patients with stroke, this finding was associated with large uncertainty in the exact estimate of the treatment effect owing to the small number of events.

In all network meta-analyses with closed loops, there was no statistically detectable inconsistency between direct and indirect evidence (see supplementary table).

\section{Discussion}

This metaepidemiological study of 16 meta-analyses including 305 randomised controlled trials with 339274 participants highlights the near absence of evidence on the comparative effectiveness of exercise and drug interventions on mortality outcomes. Existing evidence on the mortality benefits of physical activity is limited to the secondary prevention of coronary heart disease, rehabilitation after stroke, treatment of heart failure, and prevention of diabetes. Perhaps unsurprisingly, the amount of evidence on the mortality benefits of exercise is considerably smaller than that on drug interventions. There is also a clear lack of exercise and drug comparisons: trials evaluating the effectiveness of pharmacotherapy rarely included physical activity as a comparator. Albeit this considerable asymmetry in the evidence base, our analysis suggests that exercise potentially had similar effectiveness to drug interventions with two exceptions. In the case of stroke rehabilitation, exercise seemed to be more effective than drug interventions. In heart failure, diuretics outperformed all comparators, including exercise. When the drug interventions were considered together in sensitivity analyses, exercise and drug interventions did not differ in terms of their mortality benefits in all conditions except for stroke rehabilitation, where exercise interventions were associated with a reduction in the odds of mortality more than drug interventions.

\section{Geometry of existing evidence}

Evidence from randomised controlled trials on the mortality benefits of exercise is scarce. Even in treatment areas where such evidence exists, exercise trials evaluating mortality outcomes were at a disadvantage in two ways: considerably fewer trials evaluated exercise than drugs ( 57 out of 305 trials), and fewer people participated in exercise trials (14 716 out of 339274 participants).

Our findings reflect the bias against testing exercise interventions and highlight the changing landscape of medical research, which seems to increasingly favour drug interventions over strategies to modify lifestyle. The current body of medical literature largely constricts clinicians to drug options. ${ }^{48}$ This blind spot in available scientific evidence prevents prescribers and their patients from understanding the clinical circumstances where drugs might provide only modest improvement but 
exercise could yield more profound or sustainable gains in health. The lopsided nature of modern medical research may fail to detect the most effective treatment for a given condition if that treatment is not a prescription drug.

As the disparity between exercise based and drug based treatment evidence has increased, clinical practice guidelines have followed suit. ${ }^{49}{ }^{50}$ For example, although earlier versions of the United States' national cholesterol education programme guidelines advised the use of statins only after exhausting interventions for intensive lifestyle modification for the prevention of coronary heart disease in people with high cholesterol levels, ${ }^{51}$ subsequent versions progressively lowered the threshold for drug treatment and considerably expanded both the scope and the intensity of drug treatment. ${ }^{52}$

\section{Clinical practice implications of this study}

The findings of our review suggest that exercise and many drug interventions are often potentially similar in terms of their mortality benefits; exercise interventions should therefore be considered as a viable alternative to, or alongside, drug therapy. Indeed, an increasing number of experts recommend prescribing an "exercise pill" as a preventive strategy to reduce morbidity and mortality. ${ }^{53}$ According to the US Centers for Disease Control and Prevention, roughly one third of clinicians prescribe exercise in primary care ${ }^{55}$ However, as previous systematic reviews have shown, there is considerable uncertainty as to the effectiveness of primary care interventions for increasing physical activity. ${ }^{567}$ As previously recommended, primary care doctors should give brief advice to most patients about the benefits of exercise and refer patients with chronic disease to a rehabilitation programme that includes an exercise intervention. ${ }^{58}$

Previous research has shown that patients with a variety of chronic conditions can expect to improve their functional capacity as well as muscle strength by a range of exercise interventions. ${ }^{89}$ Although we considered exercise interventions as a group, a more nuanced consideration of the effectiveness of different types of physical activity is warranted. Among the four therapeutic conditions considered in our review, exercise interventions varied in terms of their type, intensity, and duration, highlighting the need to tailor exercise regimens for individual patient circumstances, such as baseline disease severity and mobility. The relative dearth of evidence leaves substantial uncertainty on which patients would benefit more from what type of exercise, and which forms of exercise may not be effective in different settings.

Complicating matters further, the evidence on the potentially harmful side effects of exercise interventions is limited. Individual trials included in the meta-analyses did not report adverse events associated with exercise interventions in a systematic manner. Nevertheless, some existing information is encouraging: according to a 10 year follow-up study of exercise based rehabilitation of patients with heart failure, there were no important adverse events requiring the interruption of any training sessions. ${ }^{59}$ Indeed, authors of the Cochrane review on exercise rehabilitation for heart failure found no evidence to suggest that such programmes cause harm. ${ }^{35}$ Similarly, authors of the Cochrane review on exercise training for stroke survivors concluded that participation in exercise training programmes seems to be safe. ${ }^{34}$ For the secondary prevention of coronary heart disease, rates of adverse events in exercise rehabilitation settings are low. ${ }^{60}$

\section{Limitations of this study}

Our findings should be interpreted in light of their limitations. Firstly, although exercise seemed to be more effective than drug interventions in stroke rehabilitation, and diuretics seemed to be more effective than exercise interventions in heart failure treatment, these findings should be interpreted with caution given the scarceness of data and the different settings involved. Although between study heterogeneity was generally low, with no statistical evidence of inconsistency between direct and indirect comparisons, it remains a possibility that potential imbalances in the distribution of unobserved or unmeasured effect modifiers across the contrasts affected the findings, ${ }^{61}$ potentially confounding the comparative estimates between drugs and exercise. Accordingly, we caution that the comparison of exercise and drug interventions should be tempered by the additional differences in patient populations across different trials.

Secondly, the definition of exercise interventions varied across the list of included trials, limiting the generalisability of our findings to different types of physical activity. Thirdly, several meta-analyses of drug interventions were outdated and it was logistically difficult to try to update so many drug meta-analyses. As a result, our review may have underestimated the magnitude of data available on drug interventions. Conversely, it is unlikely that we missed substantial randomised trial data on exercise, thus the relative dearth of evidence on exercise versus evidence on drugs may be even more prominent. Our Medline search identified only three new exercise trials, ${ }^{5962} 63$ although it captured 31 of the 54 older ones that had been included in previous meta-analyses. Based on the capture-recapture principle, we may have missed another two exercise trials only-that is, $3 \times(54-31) / 31$. Overall, the evidence on the effects of exercise is clearly outnumbered by the evidence on drugs. Moreover, if anything, there is evidence that updated meta-analyses tend to show smaller treatment effects, ${ }^{64}$ so the benefits of some drug interventions may be overrated.

Fourthly, exercise trials often included some drug interventions (although the details of drug treatments were limited), which were given similarly to both the physical therapy and the usual care arms, suggesting that the observed effect of exercise possibly represented the added benefit of exercise over and above the benefit conferred by drug interventions. This might have underestimated the effectiveness of exercise on mortality, according to the findings of recent research showing that statin treatment may decrease the fitness benefits of exercise. ${ }^{65}$

As an overview of published meta-analyses, our review also shares the limitations of the existing evidence. Previous reviews (and the randomised controlled trials on which they are based) did not always make clear the settings in which physical interventions were undertaken, how they were carried out, and potential factors responsible for the success or failure of the intervention. It is particularly important to characterise these so-called "support factors" of exercise interventions in randomised controlled trials (for example, extent of supervision, individual level versus group level activity), which would allow future researchers and practitioners to replicate successful interventions and avoid unsuccessful ones. ${ }^{66}$

\section{Future directions}

Despite its limitations, this meta-epidemiological review is the first to compare the mortality benefits of exercise and drug interventions. This comprehensive look at the existing body of evidence highlights the need to perform randomised trials on the comparative effectiveness of exercise and drug interventions. 
Given the scarcity of financial resources to fund future trials of exercise interventions, one option would be to require such evidence from pharmaceutical companies that are under increasing pressure to perform active-comparator trials for market entry. ${ }^{67}{ }^{68}$ For example, regulators should consider requiring pharmaceutical sponsors of new drugs to include exercise interventions as an active comparator arm in drug trials. In cases where drug options provide only modest benefit, patients deserve to understand the relative impact that physical activity might have on their condition.

We thank Anthony J Damico (Kaiser Family Foundation, Washington DC, USA) for his contribution to the systematic review, evaluation of the geometry of the existing evidence, and his comments on an earlier version of this paper; and Ingrid Hopper and colleagues for sharing data from their published meta-analysis.

Contributors: HN conceived the study. JPAI provided guidance during study design, protocol development, analysis, interpretation, and manuscript preparation. $\mathrm{HN}$ and Anthony J Damico identified and selected studies for inclusion in the review. HN extracted data from included meta-analyses, and Anthony J Damico checked for accuracy. $\mathrm{HN}$ did all of the statistical analyses. HN and JPAI interpreted the findings. HN drafted the paper and JPAI made revisions on subsequent drafts. Both authors read and approved the final version of the paper. $\mathrm{HN}$ is the guarantor.

\section{Funding: This study received no funding}

Competing interests: All authors have completed the ICMJE uniform disclosure form at http://www.icmje.org/coi_disclosure.pdf and declare: no support from any organisation for the submitted work; no financial relationships with any organisations that might have an interest in the submitted work in the previous three years, no other relationships or activities that could appear to have influenced the submitted work. Ethical approval: Not required.

Data sharing: Raw data used in the analysis is available on request from the corresponding author at h.naci@Ise.ac.uk.

1 Pedersen BK, Saltin B. Evidence for prescribing exercise as therapy in chronic disease. Scand J Med Sci Sports 2006;16(S1):3-63.

2 Roddy E, Zhang W, Doherty M. Aerobic walking or strengthening exercise for osteoarthritis of the knee? A systematic review. Ann Rheum Dis 2005;64:544-8.

3 Knols R, Aaronson NK, Uebelhart D, Fransen J, Aufdemkampe G. Physical exercise in cancer patients during and after medical treatment: a systematic review of randomized and controlled clinical trials. J Clin Oncol 2005;23:3830-42.

4 Fong DYT, Ho JWC, Hui BPH, Lee AM, Macfarlane DJ, Leung SSK, et al. Physical activity for cancer survivors: meta-analysis of randomised controlled trials. BMJ 2012;344:e70.

5 Sigal RJ, Kenny GP, Wasserman DH, Castaneda-Sceppa C, White RD. Physical activity/exercise and type 2 diabetes: a consensus statement from the American Diabetes Association. Diabetes Care 2006;29:1433-8.

6 Fletcher GF, Balady G, Blair SN, Blumenthal J, Caspersen C, Chaitman B, et al. Statement on exercise: benefits and recommendations for physical activity programs for all americans: a statement for health professionals by the Committee on Exercise and Cardiac Rehabilitation of the Council on Clinical Cardiology, American Heart Association. Circulation 1996;94:857-62.

7 Garcia-Aymerich J, Lange P, Benet M, Schnohr P, Anto JM. Regular physical activity reduces hospital admission and mortality in chronic obstructive pulmonary disease: a population based cohort study. Thorax 2006;61:772-8.

8 Kujala UM. Evidence for exercise therapy in the treatment of chronic disease based on at least three randomized controlled trials-summary of published systematic reviews. Scand J Med Sci Sports 2004;14:339-45.

9 Kujala UM. Evidence on the effects of exercise therapy in the treatment of chronic disease. Br J Sports Med 2009;43:550-5.

10 Byberg L, Melhus H, Gedeborg R, Sundstrom J, Ahlbom A, Zethelius B, et al. Total mortality after changes in leisure time physical activity in 50 year old men: 35 year follow-up of population based cohort. BMJ 2009;338:b688.

11 Samitz G, Egger M, Zwahlen M. Domains of physical activity and all-cause mortality: systematic review and dose-response meta-analysis of cohort studies. Int J Epidemio 2011;40:1382-400.

12 Wen CP, Wai JPM, Tsai MK, Yang YC, Cheng TYD, Lee M-C, et al. Minimum amount of physical activity for reduced mortality and extended life expectancy: a prospective cohort study. Lancet 2011;378:1244-53.

13 Blair SN. Physical inactivity: the biggest public health problem of the 21 st century. Br J Sports Med 2009;43:1-2

14 Lim SS, Vos T, Flaxman AD, Danaei G, Shibuya K, Adair-Rohani H, et al. A comparative risk assessment of burden of disease and injury attributable to 67 risk factors and risk factor clusters in 21 regions, 1990?2010: a systematic analysis for the Global Burden of Disease Study 2010. Lancet 2012;380:2224-60.

15 Department of Health. UK physical activity guidelines. DoH, 2011.
16 Townsend N, Bhatnagar P, Wickramasinghe K, Scarborough P, Foster C, Rayner M. Physical activity statistics 2012. British Heart Foundation, 2012.

17 NHS Information System. Prescriptions dispensed in the community: England, Statistics for 2001-2011. NHS Information System, 2012.

18 Naci H, Brugts JJ, Fleurence R, Tsoi B, Toor H, Ades A. Comparative benefits of statins in the primary and secondary prevention of major coronary events and all-cause mortality: a network meta-analysis of placebo-controlled and active-comparator trials. Eur J Prev Cardiol 2013:20:641-57.

19 Taylor RS, Brown A, Ebrahim S, Jolliffe J, Noorani H, Rees K, et al. Exercise-based rehabilitation for patients with coronary heart disease: systematic review and meta-analysis of randomized controlled trials. Am J Med 2004;116:682-92.

20 Higgins JP, Green S, Collaboration C. Cochrane handbook for systematic reviews of interventions: Wiley Online Library, 2008.

21 Salanti G, Higgins JP, Ades A, loannidis JP. Evaluation of networks of randomized trials. Stat Methods Med Res 2008;17:279-301.

22 Salanti G, Kavvoura FK, loannidis JP. Exploring the geometry of treatment networks. Ann Intern Med 2008; 148:544-53.

23 DerSimonian R, Laird N. Meta-analysis in clinical trials. Control Clin Trials 1986;7:177-88.

24 Lu G, Ades AE. Combination of direct and indirect evidence in mixed treatment comparisons. Stat Med 2004;23:3105-24.

25 Mills EJ, Thorlund K, loannidis JP. Demystifying trial networks and network meta-analysis. BMJ 2013;346:f2914

26 Dias S, Sutton AJ, Ades AE, Welton NJ. NICE DSU technical support document 2: a generalised linear modelling framework for pairwise and network meta-analysis of randomised controlled trials. 2011. www.nicedsu.org.uk.

27 Dias S, Sutton AJ, Ades AE, Welton NJ. Evidence synthesis for decision making 2: a generalized linear modeling framework for pairwise and network meta-analysis of randomized controlled trials. Med Decis Making 2013;33:607-17.

28 Cooper NJ, Sutton AJ, Morris D, Ades AE, Welton NJ. Addressing between-study heterogeneity and inconsistency in mixed treatment comparisons: application to stroke prevention treatments in individuals with non-rheumatic atrial fibrillation. Stat Med 2009;28:1861-81.

29 Borenstein M, Hedges LV, Higgins JPT, Rothstein HR. A basic introduction to fixed-effect and random-effects models for meta-analysis. Res Synth Method 2010;1:97-111.

30 Bucher HC, Guyatt GH, Griffith LE, Walter SD. The results of direct and indirect treatment comparisons in meta-analysis of randomized controlled trials. J Clin Epidemiol 1997;50:683-91.

31 Song F, Altman DG, Glenny AM, Deeks JJ. Validity of indirect comparison for estimating efficacy of competing interventions: empirical evidence from published meta-analyses. BMJ 2003;326:472.

32 Song F, Xiong T, Parekh-Bhurke S, Loke YK, Sutton AJ, Eastwood AJ, et al. Inconsistency between direct and indirect comparisons of competing interventions: meta-epidemiological study. BMJ 2011;343:d4909.

33 Heran BS, Chen JM, Ebrahim S, Moxham T, Oldridge N, Rees K, et al. Exercise-based cardiac rehabilitation for coronary heart disease. Cochrane Database Syst Rev 2011(7):CD001800.

34 Brazzelli M, Saunders DH, Greig CA, Mead GE. Physical fitness training for stroke patients. Cochrane Database Syst Rev 2011(11):CD003316.

35 Davies EJ, Moxham T, Rees K, Singh S, Coats AJ, Ebrahim S, et al. Exercise based rehabilitation for heart failure. Cochrane Database Syst Rev 2010(4):CD003331.

36 Hopper I, Billah B, Skiba M, Krum H. Prevention of diabetes and reduction in major cardiovascular events in studies of subjects with prediabetes: meta-analysis of randomised controlled clinical trials. Eur J Cardiovasc Prev Rehabil 2011;18:813-23.

37 Freemantle N, Cleland J, Young P, Mason J, Harrison J. $\beta$ Blockade after myocardial infarction: systematic review and meta regression analysis. BMJ 1999;318:1730-7.

38 Domanski MJ, Exner DV, Borkowf CB, Geller NL, Rosenberg Y, Pfeffer MA. Effect of angiotensin converting enzyme inhibition on sudden cardiac death in patients following acute myocardial infarction. A meta-analysis of randomized clinical trials. J Am Coll Cardiol 1999;33:598-604.

39 Collaborative meta-analysis of randomised trials of antiplatelet therapy for prevention of death, myocardial infarction, and stroke in high risk patients. BMJ 2002;324:71-86.

40 Sandercock PA, Counsell C, Kamal AK. Anticoagulants for acute ischaemic stroke. Cochrane Database Syst Rev 2008(4):CD000024.

41 Sandercock PA, Counsell C, Gubitz GJ, Tseng MC. Antiplatelet therapy for acute ischaemic stroke. Cochrane Database Syst Rev 2008(3):CD000029.

42 Wardlaw JM, Murray V, Berge E, Del Zoppo GJ. Thrombolysis for acute ischaemic stroke. Cochrane Database Syst Rev 2009(4):CD000213.

43 Flather MD, Yusuf S, Kober L, Pfeffer M, Hall A, Murray G, et al. Long-term ACE-inhibitor therapy in patients with heart failure or left-ventricular dysfunction: a systematic overview of data from individual patients. ACE-Inhibitor Myocardial Infarction Collaborative Group. Lancet 2000;355:1575-81.

44 Faris RF, Flather M, Purcell H, Poole-Wilson PA, Coats AJ. Diuretics for heart failure. Cochrane Database Syst Rev 2012;(2):CD003838.

45 Chatterjee S, Biondi-Zoccai G, Abbate A, D'Ascenzo F, Castagno D, Van Tassell B, et al. Benefits of beta blockers in patients with heart failure and reduced ejection fraction: network meta-analysis. BMJ 2013;346: 555 .

46 Heran BS, Musini VM, Bassett K, Taylor RS, Wright JM. Angiotensin receptor blockers for heart failure. Cochrane Database Syst Rev 2012;(4):CD003040

47 Berge $E$, Sandercock $P$. Anticoagulants versus antiplatelet agents for acute ischaemic stroke. Cochrane Database Syst Rev 2002(4):CD003242.

48 Moynihan R, Smith R. Too much medicine? BMJ 2002;324:859-60.

49 Getz L, Luise Kirkengen A, Hetlevik I, Romundstad S, Sigurdsson JA. Ethical dilemmas arising from implementation of the European guidelines on cardiovascular disease prevention in clinical practice. A descriptive epidemiological study. Scand J Prim Health Care 2004;22:202-8.

50 Westin S, Heath I. Thresholds for normal blood pressure and serum cholesterol. BMJ 2005;330:1461-2.

51 Summary of the second report of the National Cholesterol Education Program (NCEP) Expert Panel on Detection, Evaluation, and Treatment of High Blood Cholesterol in Adults (Adult Treatment Panel II). JAMA 1993;269:3015-23.

52 Executive Summary of The Third Report of The National Cholesterol Education Program (NCEP) Expert Panel on Detection, Evaluation, And Treatment of High Blood Cholestero In Adults (Adult Treatment Panel III). JAMA 2001;285:2486-97.

53 Khan KM, Weiler R, Blair SN. Prescribing exercise in primary care. BMJ 2011;343:d4141 


\section{What is already known on this topic}

Population level cohort studies suggest that physical activity is associated with a decreased risk of mortality A large number of randomised controlled trials show the mortality benefits of certain drug interventions

\section{What this study adds}

Evidence on the mortality benefits of drug and exercise interventions for common diseases varies widely, highlighting the paucity of data on the effectiveness of exercise interventions in randomised trials

Based on available data on the secondary prevention of coronary heart disease, stroke, heart failure, and prediabetes, physical activity is potentially as effective as many drug interventions

54 Warburton DER, Nicol CW, Bredin SSD. Prescribing exercise as preventive therapy. CMAJ 2006;174:961-74.

55 Barnes $\mathrm{P}$, Schoenborn $\mathrm{C}$. Trends in adults receiving a recommendation for exercise or other physical activity from a physician or other health professional. In: US Centers for Disease Control and Prevention, ed. 2012.

56 Pavey TG, Taylor AH, Fox KR, Hillsdon M, Anokye N, Campbell JL, et al. Effect of exercise referral schemes in primary care on physical activity and improving health outcomes: systematic review and meta-analysis. BMJ 2011;343:d6462.

57 Orrow G, Kinmonth A-L, Sanderson S, Sutton S. Effectiveness of physical activity promotion based in primary care: systematic review and meta-analysis of randomised controlled trials. BMJ 2012;344:e1389.

58 Williams NH. Promoting physical activity in primary care. BMJ 2011;343:d6615.

59 Belardinelli R, Georgiou D, Cianci G, Purcaro A. 10-year exercise training in chronic heart failure: a randomized controlled trial. J Am Coll Cardiol 2012;60:1521-8.

60 Ades PA. Cardiac rehabilitation and secondary prevention of coronary heart disease. $N$ Engl J Med 2001;345:892-902

61 Jansen JP, Naci H. Is network meta-analysis as valid as standard pairwise meta-analysis? It all depends on the distribution of effect modifiers. BMC Med 2013;11:159.

62 Davidson PM, Cockburn J, Newton PJ, Webster JK, Betihavas V, Howes L, et al. Can a heart failure-specific cardiac rehabilitation program decrease hospitalizations and improve outcomes in high-risk patients? Eur J Cardiovasc Prev Rehabil 2010;17:393-402.

63 West RR, Jones DA, Henderson AH. Rehabilitation after myocardial infarction trial (RAMIT) multi-centre randomised controlled trial of comprehensive cardiac rehabilitation in patients following acute myocardial infarction. Heart 2012;98:637-44.
64 Pereira TV, loannidis J. Statistically significant meta-analyses of clinical trials have modest credibility and inflated effects. J Clin Epidemiol 2011;64:1060-9.

65 Thompson PD, Parker B. Statins, exercise, and exercise training. J Am Coll Cardiol 2013;62:715-6.

66 Cartwright N, Hardie J. Evidence-based policy: a practical guide to doing it better. Oxford University Press, 2012.

67 Sorenson C, Naci H, Cylus J, Mossialos E. Evidence of comparative efficacy should have a formal role in European drug approvals. BMJ 2011;343:d4849.

68 Naci H, Cylus J, Vandoros S, Sato A, Perampaladas K. Raising the bar for market authorisation of new drugs. BMJ 2012;345:e4261.

\section{Accepted: 22 August 2013}

\section{Cite this as: BMJ 2013;347:f5577}

This is an Open Access article distributed in accordance with the Creative Commons Attribution Non Commercial (CC BY-NC 3.0) license, which permits others to distribute, remix, adapt, build upon this work non-commercially, and license their derivative works on different terms, provided the original work is properly cited and the use is non-commercial. See: http://creativecommons.org/licenses/by-nc/3.0/. 


\section{Tables}

Table 1| Findings of network meta-analyses: comparative effects of exercise and drug interventions on mortality outcomes in coronary heart disease. Values are odds ratios $(95 \%$ credible intervals)

\begin{tabular}{lccccc}
\multirow{2}{*}{ Intervention } & \multicolumn{5}{c}{ Comparator drugs } \\
\cline { 2 - 6 } Exercise & $1.08(0.90$ to 1.30$)$ & $1.05(0.87$ to 1.25$)$ & $1.08(0.87$ to 1.33$)$ & $1.07(0.88$ to 1.30$)$ \\
\hline Statins & - & $0.97(0.85$ to 1.10$)$ & $0.99(0.84$ to 1.18$)$ & $0.99(0.85$ to 1.15$)$ \\
\hline$\beta$ blockers & - & - & $1.03(0.87$ to 1.21$)$ & $1.02(0.89$ to 1.17$)$ \\
\hline ACE inhibitors & - & - & - & $0.99(0.83$ to 1.19$)$ \\
\hline
\end{tabular}

$\mathrm{ACE}=$ angiotensin converting enzyme.

Estimates lower than 1.00 favour row defining intervention. 
Table 2| Findings of network meta-analyses: comparative effects of exercise and drug interventions on mortality outcomes in stroke. Values are odds ratios ( $95 \%$ credible intervals)

Comparator drugs

Intervention Anticoagulants Antiplatelets

Exercise $\quad 0.09(0.01$ to 0.70$) \quad 0.10(0.01$ to 0.62$)$

Anticoagulants $\quad-\quad 1.11(1.00$ to 1.21$)$

Estimates lower than 1.00 favour row defining intervention. 
Table 3| Findings of network meta-analyses: comparative effects of exercise and drug interventions on mortality outcomes in heart failure. Values are odds ratios ( $95 \%$ credible intervals)

\begin{tabular}{lccccc} 
& \multicolumn{5}{c}{ Comparator drugs } \\
\cline { 2 - 6 } Intervention & ACE inhibitors & Diuretics & $\boldsymbol{\beta}$ blockers & ARBs \\
Exercise & $0.89(0.59$ to 1.23$)$ & $4.11(1.17$ to 24.76$)$ & $1.11(0.82$ to 1.46$)$ & $0.86(0.62$ to 1.16$)$ \\
\hline ACE inhibitors & - & $4.66(1.32$ to 28.21$)$ & $1.24(0.96$ to 1.71$)$ & $0.96(0.78$ to 1.27$)$ \\
\hline Diuretics & - & - & $0.27(0.04$ to 0.93$)$ & 0.21 (0.03 to 0.73$)$ \\
\hline$\beta$ blockers & - & - & - & 0.77 (0.62 to 0.98$)$ \\
\hline
\end{tabular}

ACE=angiotensin converting enzyme; $\mathrm{ARBs}=$ angiotensin receptor blockers.

Estimates lower than 1.00 favour row defining intervention. 
Table 4| Findings of network meta-analyses: comparative effects of exercise and drug interventions on mortality outcomes in prediabetes. Values are odds ratios (95\% credible intervals)

\begin{tabular}{lccccc} 
& \multicolumn{5}{c}{ Comparator drugs } \\
\cline { 2 - 6 } Intervention & AGls & Biguanides & ACE inhibitors & Glinides \\
Exercise & $0.22(0.02$ to 1.18$)$ & $2.67(0.41$ to 36.39$)$ & $0.73(0.14$ to 1.96$)$ & $0.69(0.10$ to 2.52$)$ \\
\hline AGls & - & $13.39(0.99$ to 519.75$)$ & $3.26(0.42$ to 43.94$)$ & $3.06(0.33$ to 48.01$)$ \\
\hline Biguanides & - & - & $0.26(0.01$ to 1.85$)$ & $0.25(0.01$ to 2.08$)$ \\
\hline ACE inhibitors & - & - & - & $0.93(0.18$ to 5.49$)$ \\
\hline
\end{tabular}

AGIs=a glucosidase inhibitors; $A C E=$ angiotensin converting enzyme.

Estimates lower than 1.00 favour row defining intervention. 
Table 5| Findings of network meta-analyses: comparative effects of exercise and all drug interventions on mortality outcomes in coronary heart disease, stroke, heart failure, and prediabetes

\begin{tabular}{lc} 
Treatment area & Odds ratio $(\mathbf{9 5 \%}$ Crl) drugs vexercise \\
Coronary heart disease & $0.94(0.80$ to 1.11$)$ \\
\hline Stroke & $8.66(1.28 \text { to } 245.10)^{*}$ \\
\hline Heart failure & $0.99(0.73$ to 1.36$)$ \\
\hline Prediabetes & $1.43(0.81$ to 3.11$)$ \\
\hline
\end{tabular}

Estimates lower than 1.00 favour drug interventions, estimates higher than 1.00 favour exercise.

*Odds ratio was associated with considerable uncertainty owing to extremely small numbers of events in exercise trials. 


\section{Figures}

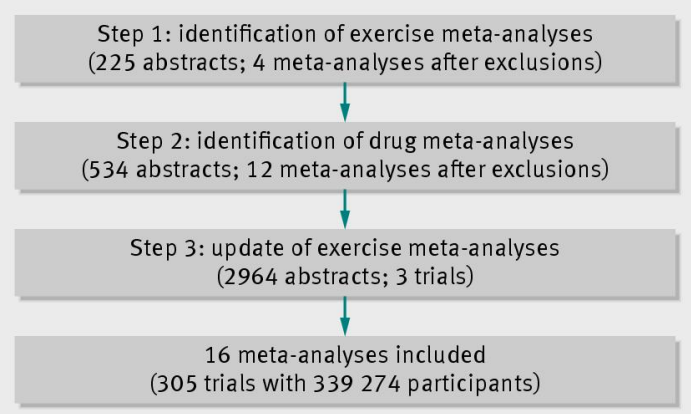

Fig 1 Steps involved in study identification and selection

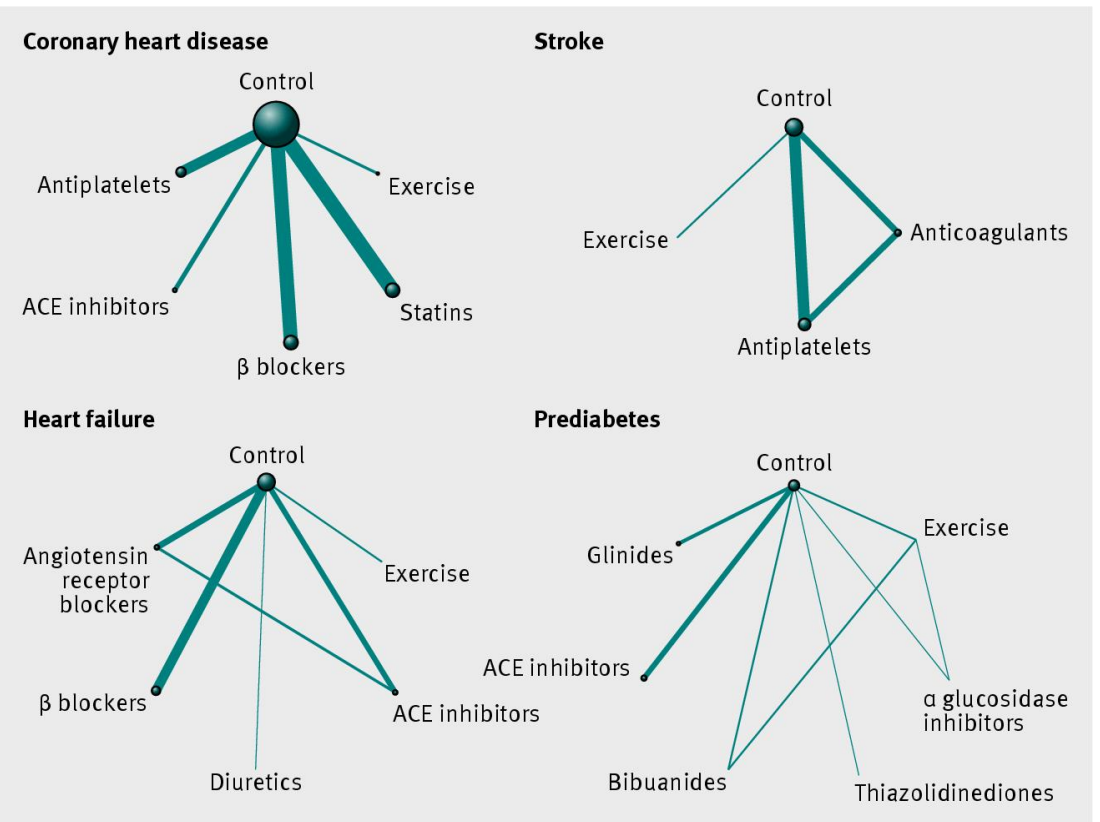

Fig 2 Network of available comparisons between exercise and individual drug interventions in coronary heart disease, stroke, heart failure, and prediabetes. Size of node is proportional to number of trial participants, and thickness of line connecting nodes is proportional to number of participants randomised in trials directly comparing the two treatments. $\mathrm{ACE}=$ angiotensin converting enzyme 


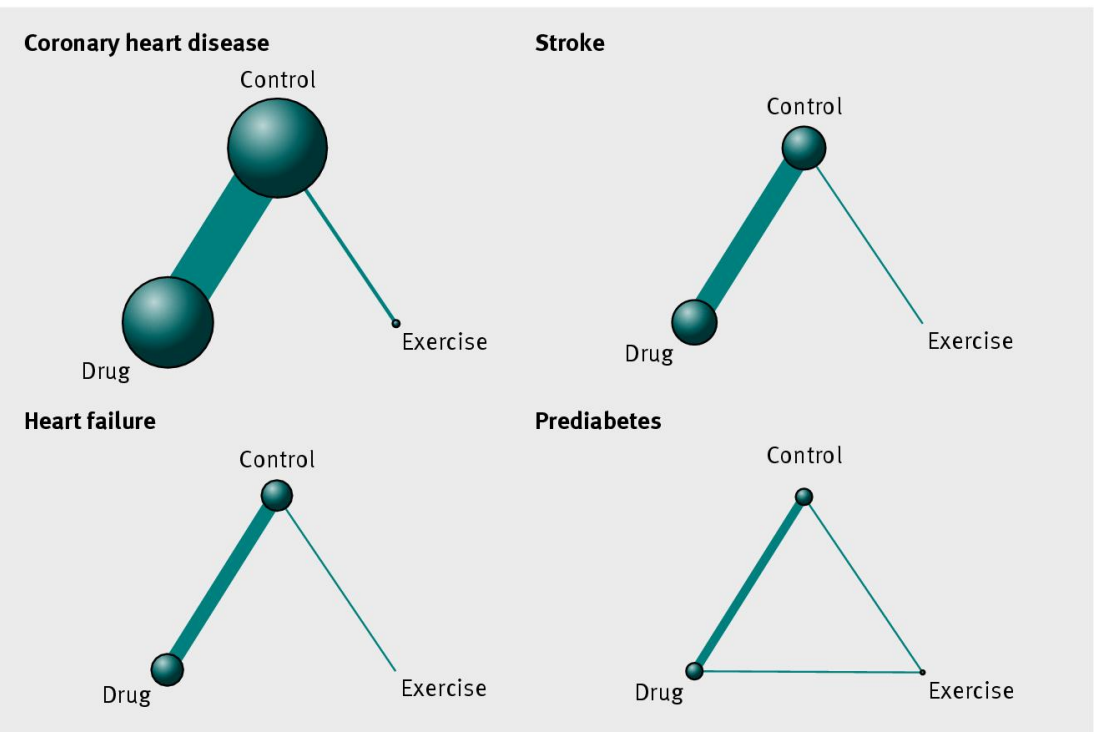

Fig 3 Network of available comparisons between exercise and all drug interventions in coronary heart disease, stroke, heart failure, and prediabetes. Size of node is proportional to number of trial participants, and thickness of line connecting nodes is proportional to number of participants randomised in trials directly comparing the two treatments

\begin{tabular}{|c|c|c|}
\hline Study & $\begin{array}{l}\text { Odds ratio } \\
(95 \% \mathrm{Crl})\end{array}$ & $\begin{array}{l}\text { Odds ratio } \\
(95 \% \mathrm{Crl})\end{array}$ \\
\hline \multicolumn{3}{|l|}{ Coronary heart disease } \\
\hline Exercise & $\rightarrow$ & $0.89(0.76$ to 1.04$)$ \\
\hline Statins & $=$ & $0.82(0.75$ to 0.90$)$ \\
\hline$\beta$ blockers & - & 0.85 (0.78 to 0.92$)$ \\
\hline ACE inhibitors & - & $0.83(0.72$ to 0.96$)$ \\
\hline Antiplatelets & $=$ & $0.83(0.74$ to 0.93$)$ \\
\hline \multicolumn{3}{|l|}{ Stroke } \\
\hline Exercise & - & 0.09 (0.01 to 0.72$)$ \\
\hline Anticoagulants & & $1.03(0.93$ to 1.12$)$ \\
\hline Antiplatelets & , & $0.93(0.85$ to 1.01$)$ \\
\hline \multicolumn{3}{|l|}{ Heart disease } \\
\hline Exercise & $\rightarrow$ & $0.79(0.59$ to 1.00$)$ \\
\hline ACE inhibitors & $\rightarrow$ & $0.88(0.69$ to 1.16$)$ \\
\hline Diuretics & - & $0.19(0.03$ to 0.66$)$ \\
\hline$\beta$ blockers & $\rightarrow$ & $0.71(0.61$ to 0.80$)$ \\
\hline Angiotensin receptor blockers & $\rightarrow$ & 0.92 (0.74 to 1.09$)$ \\
\hline \multicolumn{3}{|l|}{ Prediabetes } \\
\hline Exercise & - & $0.67(0.22$ to 1.27$)$ \\
\hline a glucosidase inhibitors & & $3.03(0.51$ to 34.87$)$ \\
\hline \multicolumn{3}{|l|}{ Thiazolidinediones* } \\
\hline Biguanides & & $0.25(0.02$ to 1.46$)$ \\
\hline ACE inhibitors & & $0.93(0.37$ to 2.59$)$ \\
\hline Glinides & & $0.99(0.25$ to 3.93$)$ \\
\hline 0.01 & 0.1 & 0 \\
\hline
\end{tabular}

Fig 4 Findings of network meta-analysis: effects of exercise and drug interventions compared with control on mortality outcomes in coronary heart disease, stroke, heart failure, and prediabetes. Results shown are odds ratios and $95 \%$ credible intervals. Odds ratios lower than 1.00 favour intervention compared with control. ACE=angiotensin converting enzyme. *Number of data points for thiazolidinediones was insufficient to obtain an estimate of odds ratio compared with control 